\title{
Extracellular PBEF/NAMPT/visfatin activates pro-inflammatory signalling in human vascular smooth muscle cells through nicotinamide phosphoribosyltransferase activity
}

\author{
T. Romacho • V. Azcutia • M. Vázquez-Bella •

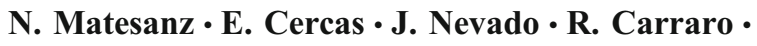 \\ L. Rodríguez-Mañas · C. F. Sánchez-Ferrer • C. Peiró
}

Received: 25 June 2009 / Accepted: 30 July 2009 /Published online: 29 August 2009

(C) Springer-Verlag 2009

\begin{abstract}
Aims/hypothesis Extracellular pre-B cell colony-enhancing factor/nicotinamide phosphoribosyltransferase/visfatin (ePBEF/NAMPT/visfatin) is an adipocytokine, whose circulating levels are enhanced in metabolic disorders, such as diabetes mellitus and obesity. Here, we explored the ability of ePBEF/NAMPT/visfatin to promote vascular inflammation, as a condition closely related to atherothrombotic diseases. We specifically studied the ability of PBEF/ NAMPT/visfatin to directly activate pathways leading to inducible nitric oxide synthase (iNOS) induction in cultured
\end{abstract}

T. Romacho $\cdot$ V. Azcutia $\cdot$ M. Vázquez-Bella $\cdot$ N. Matesanz

E. Cercas $\cdot$ C. F. Sánchez-Ferrer $\cdot$ C. Peiró $(\square)$

Departamento de Farmacología y Terapéutica,

Facultad de Medicina, Universidad Autónoma de Madrid,

Arzobispo Morcillo, 4,

28029 Madrid, Spain

e-mail: concha.peiro@uam.es

\section{J. Nevado}

Instituto de Genética Médica y Molecular (INGEMM),

Hospital Universitario La Paz,

Madrid, Spain

R. Carraro

Servicio de Endocrinología, Hospital Universitario de La Princesa y Universidad Autónoma de Madrid,

Madrid, Spain

\section{Rodríguez-Mañas}

Unidad de Investigación y Servicio de Geriatría,

Hospital Universitario de Getafe,

Getafe, Spain human aortic smooth muscle cells, as well as the mechanisms involved.

Methods iNOS levels and extracellular signal-regulated kinase (ERK) 1/2 activity were determined by western blotting. Nuclear factor (NF)- $\mathrm{kB}$ activity was assessed by electrophoretic mobility shift assay.

Results ePBEF/NAMPT/visfatin $(10-250 \mathrm{ng} / \mathrm{ml})$ induced iNOS in a concentration-dependent manner. At a submaximal concentration $(100 \mathrm{ng} / \mathrm{ml})$, ePBEF/NAMPT/visfatin time-dependently enhanced iNOS levels up to $18 \mathrm{~h}$ after stimulation. Over this time period, ePBEF/NAMPT/visfatin elicited a sustained activation of NF- $\mathrm{KB}$ and triggered a biphasic ERK 1/2 activation. By using the respective ERK $1 / 2$ and NF-KB inhibitors, PD98059 and pyrrolidine dithiocarbamate, we established that iNOS induction by ePBEF/NAMPT/visfatin required the consecutive upstream activation of ERK $1 / 2$ and NF- $\mathrm{KB}$. The pro-inflammatory action of ePBEF/NAMPT/visfatin was not prevented by insulin receptor blockade. However, exogenous nicotinamide mononucleotide, the product of NAMPT activity, mimicked NF- $\mathrm{KB}$ activation and iNOS induction by ePBEF/NAMPT/visfatin, while the NAMPT inhibitor APO866 prevented the effects of ePBEF/NAMPT/visfatin on iNOS and NF-kB.

Conclusions/interpretation Through its intrinsic NAMPT activity, ePBEF/NAMPT/visfatin appears to be a direct contributor to vascular inflammation, a key feature of atherothrombotic diseases linked to metabolic disorders.

Keywords Adipocytokine - Atherosclerosis · Inducible nitric oxide synthase · Inflammation . PBEF/NAMPT/visfatin · Vascular smooth muscle 


$\begin{array}{ll}\begin{array}{l}\text { Abbreviations } \\ \text { ePBEF/ }\end{array} & \text { Extracellular PBEF/NAMPT/ } \\ \text { NAMPT/visfatin } & \text { visfatin } \\ \text { ERK } 1 / 2 & \begin{array}{l}\text { Extracellular-signal regulated } \\ \text { kinase } 1 / 2\end{array} \\ \text { HASMC } & \text { Human aortic smooth muscle cells } \\ \text { iNOS } & \text { Inducible nitric oxide synthase } \\ \text { NAMPT } & \text { Nicotinamide phosphoribosyltransferase } \\ \text { NF-KB } & \text { Nuclear factor-KB } \\ \text { NMN } & \text { Nicotinamide mononucleotide } \\ \text { PBEF } & \text { Pre-B cell colony-enhancing factor } \\ \text { PDTC } & \text { Pyrrolidine dithiocarbamate }\end{array}$

\section{Introduction}

Extracellular pre-B cell colony-enhancing factor/nicotinamide phosphoribosyltransferase/visfatin (ePBEF/NAMPT/ visfatin) has been identified as an adipocytokine preferentially released by visceral fat [1]. Whereas enhanced circulating ePBEF/NAMPT/visfatin levels have been reported in patients affected by metabolic disorders, such as diabetes mellitus, obesity or the metabolic syndrome $[2,3]$, the pathophysiological relevance of ePBEF/NAMPT/ visfatin remains poorly understood. Enhanced circulating levels of ePBEF/NAMPT/visfatin have been positively associated not only with increased plasma levels of inflammatory markers [2], but also with vascular damage and endothelial dysfunction [4, 5]. An emerging hypothesis is that ePBEF/NAMPT/visfatin might directly promote vascular inflammation, therefore representing a link between metabolic disorders and atherothrombotic diseases exhibiting a chronic pro-inflammatory background, including atherosclerosis and CHD.

Despite this latter hypothesis, the ability of ePBEF/ NAMPT/visfatin to exert direct pro-inflammatory effects on the vasculature remains largely unknown. To address this question, we used cultured human aortic smooth muscle cells (HASMC) to investigate the ability of ePBEF/ NAMPT/visfatin to activate the extracellular-signal regulated kinase (ERK) 1/2-nuclear factor (NF)- $k B$-inducible nitric oxide synthase (iNOS) axis, whose activation is associated with vascular damage, inflammation and cardiovascular complications of diabetes [6]. We further aimed to identify the mechanism mediating the potential proinflammatory action of ePBEF/NAMPT/visfatin in human vascular smooth muscle cells.

\section{Methods}

Materials Culture plastic ware was from TPP (Tragadingen, Switzerland). DMEM, FCS and trypsin-EDTA were from Biological Industries (Beit-Hamek, Israel). Both human recombinant $\mathrm{PBEF} / \mathrm{NAMPT} /$ visfatin and IL-1 $\beta$ were purchased from Peprotech (London, UK), with an endotoxin level $<0.1 \mathrm{ng} / \mu \mathrm{g}$. APO866 was kindly donated by Topotarget (Lausanne, Switzerland). The blocking mouse monoclonal antibody (Ab-3, clone 47-9) against the human insulin receptor ( $\alpha$-subunit) was purchased from Labvision (Fremont, CA, USA). Pyrrolidine dithiocarbamate (PDTC), ERK 1/2 inhibitor PD98059, human recombinant insulin, nicotinamide mononucleotide (NMN) and, unless otherwise stated, all other reagents were purchased from Sigma (St Louis, MO, USA).

Cell culture HASMC were obtained by enzymatic dissociation from the aortas of five organ donors, conforming with the principles outlined in the Declaration of Helsinki and accordingly to Spanish legal regulations [7]. HASMC were routinely cultured in DMEM supplemented with $10 \%$ (vol./vol.) FCS and antibiotics [7]. At confluence, HASMC were deprived of serum for $24 \mathrm{~h}$ prior to the addition of the different test compounds in serum-free medium supplemented with $0.1 \%$ (wt/vol.) BSA. Cultures between passages three and ten were used.

Western blotting Proteins $(15 \mu \mathrm{g})$ from HASMC lysates were separated by SDS-PAGE, transferred to nitrocellulose membranes (Whatman, Maidstone, UK) and probed with a primary polyclonal antibody against iNOS (dilution 1/10,000; Transduction Laboratories, Lexington, KY, USA), followed by incubation with a horseradish peroxidase-conjugated secondary antibody (dilution 1/10,000; Chemicon, Temecula, CA, USA), as described previously [7]. Immunoreactive bands were detected by enhanced chemiluminescence (GE Healthcare, Uppsala, Sweden) and quantified using NIH ImageJ software (http://rsb.info.nih.gov/ij/, accessed 8 July 2005). The membranes were stripped and reprobed with an anti- $\alpha$ tubulin primary antibody (Sigma; dilution 1/10,000) to ensure equal loading.

ERK $1 / 2$ activity was determined by western blotting using specific antibodies against both the phosphorylated (activated) form of ERK 1/2 (dilution 1/1,000; Cell Signaling Technology, Danvers, MA, USA) and total ERK 1/2 (dilution 1/1,000; Santa Cruz Biotechnology, Santa Cruz, CA, USA). ERK $1 / 2$ activity was expressed as the phosphorylated ERK 1/2:total ERK 1/2 ratio.

Indirect immunofluorescence In HASMC, iNOS was visualised by indirect immunofluorescence, as previously described [7].

Nuclear extracts and electrophoretic mobility shift assay $\mathrm{Nu}-$ clear extracts from HASMC cultures were prepared as 
described before [7]. A commercial oligonucleotide (Promega, Madison, WI, USA) encoding the NF-kB consensus sequence (5'-AGTTGAGGGGACTTTC CCAGGC-3') was $5^{\prime}$-end labelled using $\left[\gamma^{3}{ }^{32} \mathrm{P}\right]$ ATP and T4 polynucleotide kinase (Promega) and purified using MicroSpin G-25 columns (GE Healthcare, Chicago, IL, USA). For binding reactions, nuclear extracts $(5 \mu \mathrm{g})$ were incubated on ice for $15 \mathrm{~min}$ in a reaction buffer (40 mmol/l HEPES [pH 7.0], $140 \mathrm{mmol} / \mathrm{l} \mathrm{NaCl}$, $5 \mathrm{mmol} / \mathrm{l}$ dithiothreitol, $10 \mu \mathrm{g} / \mathrm{ml}$ BSA, $0.01 \%$ [vol./vol.] Nonidet P-40, 4\% [wt/vol.] Ficoll and $0.05 \mu \mathrm{g} / \mathrm{ml}$ poly [dI-dC]). After addition of the labelled oligonucleotide $(\sim 50,000 \mathrm{cpm})$ the reaction mix was further incubated for $20 \mathrm{~min}$ at room temperature. For competition experiments a 100 -fold excess of unlabelled doubled-stranded oligonucleotides was added to the binding reaction. DNA-protein complexes were resolved on $4 \%$ (wt/vol.) non-denaturing polyacrylamide gels in $0.5 \times \mathrm{TBE}(45 \mathrm{mmol} / \mathrm{l}$ TRIS-borate, $1 \mathrm{mmol} / 1 \mathrm{EDTA}, \mathrm{pH} 8.0$ ) at $4^{\circ} \mathrm{C}$. Gels were dried and exposed to autoradiography at $-80^{\circ} \mathrm{C}$.

Statistical analysis Results are expressed as means \pm SEM of at least three independent experiments. Statistical analysis was performed using ANOVA for curves or Student's $t$ test for data points, with the level of significance chosen at $p<0.05$.

\section{Results}

ePBEF/NAMPT/visfatin induces iNOS in HASMC through an ERK 1/2- and NK- $\kappa B$-dependent mechanism In HASMC exposed to ePBEF/NAMPT/visfatin (10-250 ng/ml) for $18 \mathrm{~h}$, a concentration-dependent increase in the levels of the proinflammatory enzyme iNOS was observed, with a threshold concentration of $50 \mathrm{ng} / \mathrm{ml}$ (Fig. 1a). Figure $1 \mathrm{~b}$ depicts the time course of iNOS induction by a sub-maximal concentration of ePBEF/NAMPT/visfatin $(100 \mathrm{ng} / \mathrm{ml})$ : iNOS levels were significantly enhanced $8 \mathrm{~h}$ after the addition of the adipocytokine and peaked at $18 \mathrm{~h}$, with no further induction of iNOS observed at $24 \mathrm{~h}(96.6 \pm 12.1 \%$ of the levels achieved at $18 \mathrm{~h}$; results from three independent experiments). The pro-inflammatory adipocytokine IL-1 $\beta(10 \mathrm{ng} / \mathrm{ml})$ was used as a well-known positive control of iNOS induction $(3.3 \pm 0.3$-fold over basal cultures; $p<0.05$, results from five independent experiments, Fig. 1b). The induction of iNOS by ePBEF/ NAMPT/visfatin $(250 \mathrm{ng} / \mathrm{ml})$ or IL-1 $\beta(10 \mathrm{ng} / \mathrm{ml})$ in HASMC was visualised by indirect immunofluorescence (Fig. 1c).

In vascular smooth muscle, the induction of iNOS by IL$1 \beta$ is regulated through the activation of the transcription factor NF- $\mathrm{KB}$, which in turn requires the upstream activation of the mitogen-activated protein kinase ERK 1/2 [8]. In HASMC, ePBEF/NAMPT/visfatin $(100 \mathrm{ng} / \mathrm{ml})$ elicited a gradual increase in NF- $\mathrm{KB}$ binding activity from 2 to $18 \mathrm{~h}$, when the maximal activation was attained (Fig. 1d). At $24 \mathrm{~h}, \mathrm{NF}-\mathrm{kB}$ activity was about $50 \%$ of that achieved at $18 \mathrm{~h}$, although it remained enhanced compared with basal levels (Fig. 1d). IL-1 $\beta$ yielded a $2.7 \pm 0.4$-fold increase over basal NF- $\mathrm{kB}$ activity after $1 \mathrm{~h}$ of treatment $(p<0.05$; results from three independent experiments). The addition of a 100 -fold excess of cold probe blunted the formation of the binding complexes, demonstrating their specificity (data not shown).

In addition, ePBEF/NAMPT/visfatin (100 $\mathrm{ng} / \mathrm{ml})$ triggered a biphasic time-dependent activation of ERK 1/2 in HASMC: at times $<1 \mathrm{~h}$, a transient increase in the phosphorylated ERK 1/2:total ERK 1/2 ratio was observed at $10 \mathrm{~min}$, which decayed to basal levels between $30 \mathrm{~min}$ and $1 \mathrm{~h}$ (Fig. 2a). Later on, a gradual and sustained activation of ERK $1 / 2$ was observed up to $18 \mathrm{~h}$ (Fig. 2a). The activation of ERK $1 / 2$ by ePBEF/ NAMPT/visfatin (10-250 $\mathrm{ng} / \mathrm{ml})$ was concentrationdependent, as shown in Fig. 2b. IL-1 $\beta$ elicited a $3.2 \pm$ 0.7 -fold increase over basal ERK $1 / 2$ activity after $18 \mathrm{~h}$ of treatment $(p<0.05$; results from three independent experiments).

We next assessed the implication of both NF-KB and ERK $1 / 2$ in ePBEF/NAMPT/visfatin-mediated iNOS induction in HASMC. The co-incubation of ePBEF/ NAMPT/visfatin $(100 \mathrm{ng} / \mathrm{ml})$ with either the ERK 1/2 inhibitor PD98059 $(30 \mu \mathrm{mol} / \mathrm{l})$ or the NF- $\mathrm{KB}$ inhibitor PDTC $(100 \mu \mathrm{mol} / \mathrm{l})$ suppressed the induction of iNOS by the adipocytokine (Fig. 2c). Neither PD98059 nor PDTC alone modified basal iNOS levels (data not shown). Furthermore, PD98059 abolished NF-KB activation triggered by PBEF/NAMPT/visfatin (Fig. 2d), highlighting the role of ERK $1 / 2$ in NF-KB activation by $\mathrm{PBEF} /$ NAMPT/visfatin.

The pro-inflammatory action of ePBEF/NAMPT/visfatin in $H A S M C$ is not mediated by the insulin receptor To gain insight into the mechanisms mediating the proinflammatory action of ePBEF/NAMPT/visfatin in HASMC, we next explored the role of the insulin receptor, as ePBEF/NAMPT/visfatin was initially proposed as an insulin mimetic [1]. For this purpose, we used an antibody that binds the $\alpha$-subunit of the insulin receptor and blocks the receptor activation $[9,10]$ and examined NF-KB activation as a key molecule regulating inflammationrelated genes. HASMC pre-incubation for $2 \mathrm{~h}$ with the blocking antibody did not affect NF- $\mathrm{KB}$ activation by ePBEF/NAMPT/visfatin (100 ng/ml) after $18 \mathrm{~h}$ (Fig. 3), and did not modify the effect of IL-1 $\beta(10 \mathrm{ng} / \mathrm{ml})$, used as a negative control (Fig. 3). Contrarily, the antibody 
Fig. 1 ePBEF/NAMPT/visfatin stimulates iNOS induction and $\mathrm{NF}-\mathrm{KB}$ binding activity in HASMC. a HASMC were challenged with increasing concentrations of visfatin $(10-250 \mathrm{ng} / \mathrm{ml})$ and $18 \mathrm{~h}$ later iNOS levels were determined by western blotting. $* p<0.05$ vs cultures without visfatin. A representative gel is shown at the top. b Time course of iNOS induction by visfatin (100 ng/ml). $\dagger p<0.05$ vs time 0 . A representative gel is shown at the top, in which the effect of IL-1 $\beta(10 \mathrm{ng} / \mathrm{ml})$, used as a positive control for iNOS induction, is shown. c Immunolocalisation of iNOS by indirect immunofluorescence in HASMC cultures untreated (basal) or treated with visfatin $(250 \mathrm{ng} / \mathrm{ml})$ or IL-1 $\beta(10 \mathrm{ng} / \mathrm{ml})$ for $18 \mathrm{~h}$. Nuclei were counterstained with DAPI. Magnification $\times 400$. $d$ Time course of $\mathrm{NF}-\kappa \mathrm{B}$ activation by visfatin (100 ng/ml). $₫ p<0.05$ vs time 0 . A representative gel is shown on the right. Means \pm SEM of at least three independent experiments a
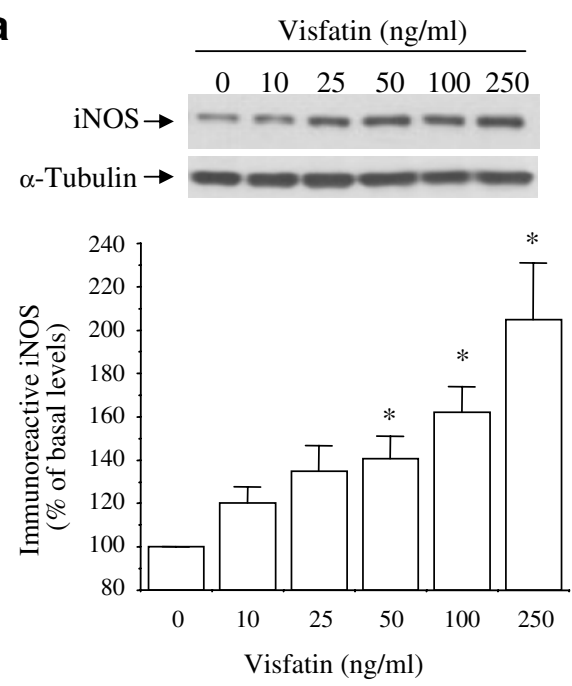

C
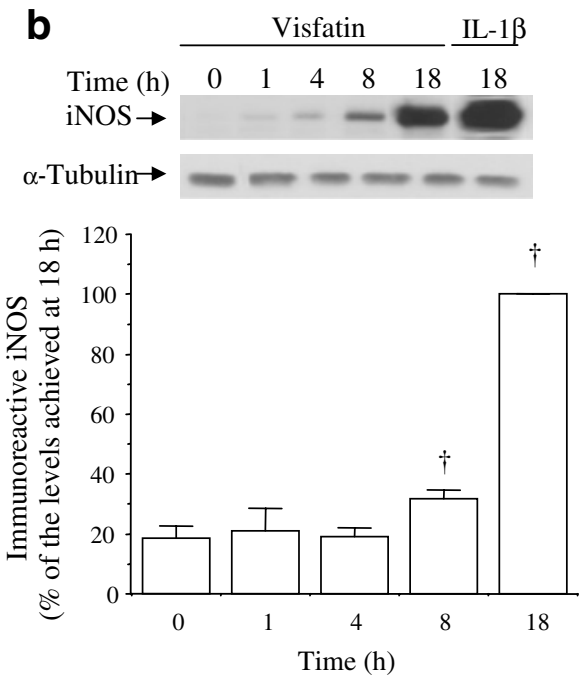

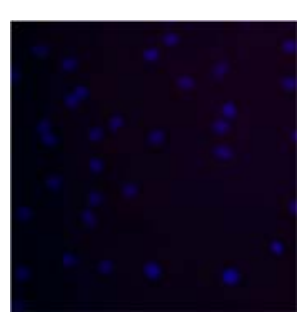

Basal

d

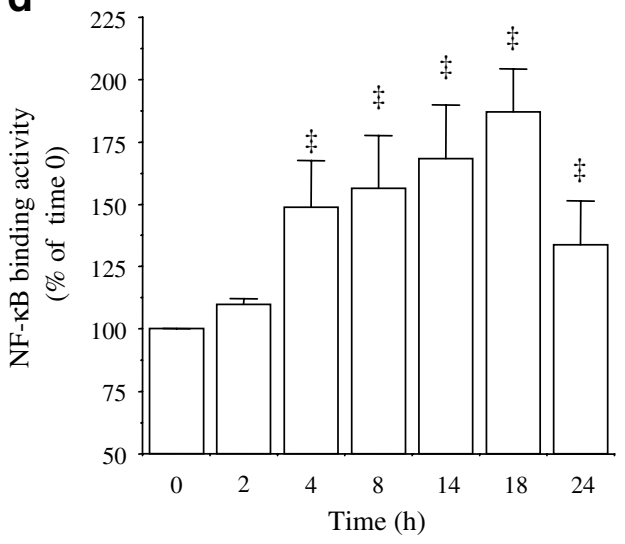

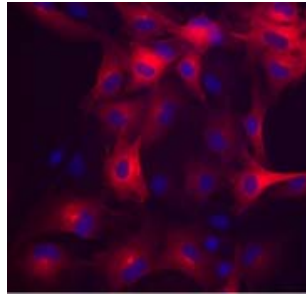

IL-1 $\beta$

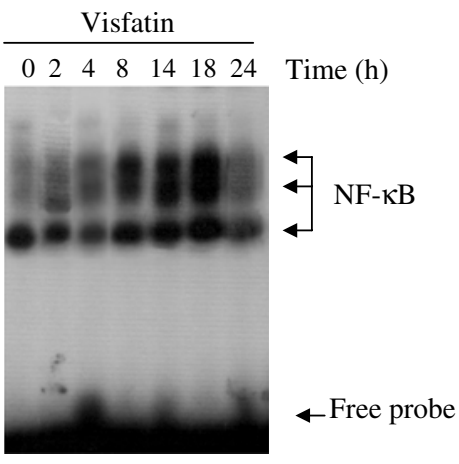

significantly reduced $\mathrm{NF}-\mathrm{KB}$ activation by insulin (10 nmol/l), used as a positive control of the receptor activation (Fig. 3).

\section{NAMPT enzymatic activity mediates the pro-inflammatory} action of ePBEF/NAMPT/visfatin in HASMC As it has been reported that $\mathrm{ePBEF} / \mathrm{NAMPT} /$ visfatin may exert its cellular actions through its intrinsic NAMPT enzymatic activity [11], we first explored whether NMN, the product of NAMPT activity and a precursor of NAD [12], could mimic the pro-inflammatory effect of PBEF/NAMPT/ visfatin in HASMC. Figure $4 \mathrm{a}$ shows that exogenous NMN $(100 \mu \mathrm{mol} / \mathrm{l})$ stimulated NF-KB activity to a similar extent as ePBEF/NAMPT/visfatin (100 ng/ml). NMN (100 $\mu \mathrm{mol} / \mathrm{l})$ also triggered iNOS induction in HASMC, which was not significantly different from that achieved by $100 \mathrm{ng} / \mathrm{ml}$ ePBEF/NAMPT/visfatin (Fig. 4b).

We next assessed the impact of NAMPT activity inhibition on the pro-inflammatory action of ePBEF/ NAMPT/visfatin. As shown in Fig. 4c, the activation of NF- $\kappa$ B elicited by ePBEF/NAMPT/visfatin $(100 \mathrm{ng} / \mathrm{ml})$ in HASMC was completely abolished by co-incubation with the NAMPT activity inhibitor APO866 (100 nmol/l) [13]. Analogously, APO866 (100 nmol/1) blocked iNOS induction by $100 \mathrm{ng} / \mathrm{ml}$ ePBEF/NAMPT/visfatin (Fig. 4d). APO866 itself did not modify NF- $\mathrm{B}$ activity or iNOS 
Fig. 2 iNOS induction by ePBEF/NAMPT/visfatin is dependent on ERK $1 / 2$ and NF- $\mathrm{KB}$ sequential activation. a Time course of ERK $1 / 2$ activation by visfatin $(100 \mathrm{ng} / \mathrm{ml})$. ERK $1 / 2$ activity was expressed as the phosphorylated ERK (p-ERK) 1/2:total ERK $1 / 2$ ratio. ${ }^{*} p<0.05$ vs time 0 . A representative gel is shown at the top. b Concentration-dependent activation of ERK $1 / 2$ after $18 \mathrm{~h}$ of stimulation with visfatin $(10$ $250 \mathrm{ng} / \mathrm{ml}) . \dagger p<0.05$ vs cultures without visfatin. A representative gel is shown at the top. c iNOS levels in HASMC exposed for $18 \mathrm{~h}$ to visfatin $(100 \mathrm{ng} / \mathrm{ml})$, alone or in the presence of the ERK 1/2 inhibitor PD98059 $(30 \mu \mathrm{mol} / 1)$ or the NF- $\mathrm{KB}$ inhibitor PDTC $(100 \mu \mathrm{mol} / 1)$. $\$ p<0.05$ vs visfatin alone. A representative immunoblot is shown at the top. d Visfatin-induced NF- $\mathrm{kB}$ binding activity is dependent on ERK $1 / 2$ activity. NF-kB activity was determined in HASMC exposed for $18 \mathrm{~h}$ to visfatin $(100 \mathrm{ng} / \mathrm{ml})$ alone or in the presence of PD98059 (30 $\mu \mathrm{mol} / 1) . \ddagger p<0.05$ vs visfatin alone. Means \pm SEM of at least three independent experiments a
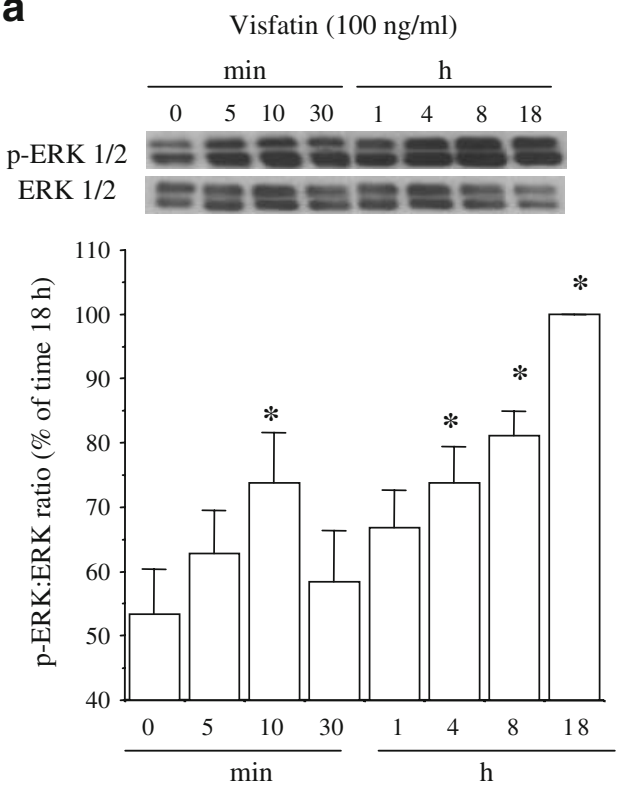

C

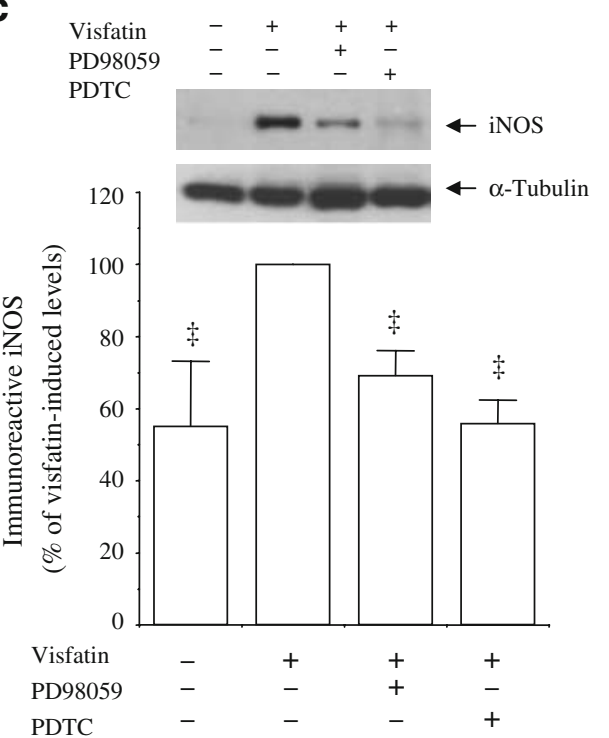

b
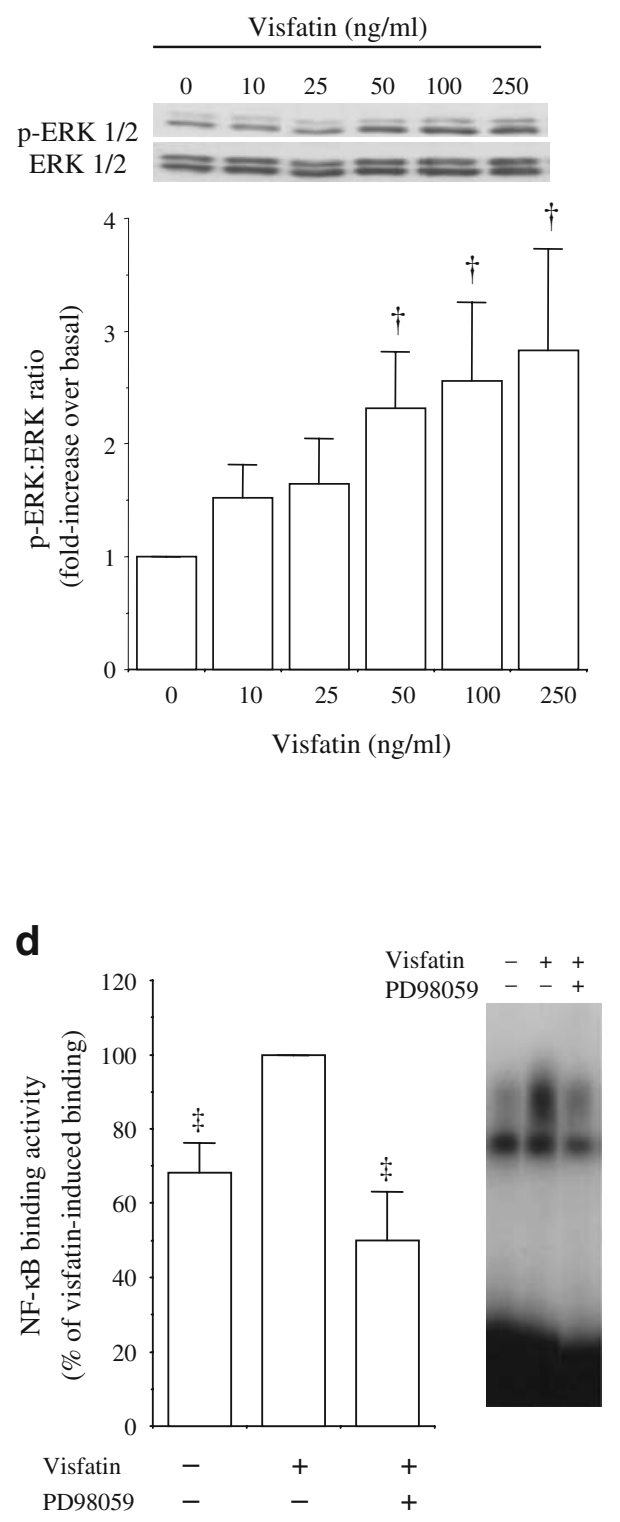

levels in non-stimulated HASMC (Fig. 4c,d). It is worth noting the NAMPT substrate nicotinamide is contained in the culture medium of HASMC (DMEM).

\section{Discussion}

In the present study, we have demonstrated the ability of the adipocytokine PBEF/NAMPT/visfatin to exert a direct activation of pro-inflammatory signals in human vascular smooth muscle. Indeed, ePBEF/NAMPT/visfatin upregulated the levels of iNOS, a nitric oxide- and peroxynitriteforming enzyme that plays a key role in vascular inflammation and endothelial dysfunction, and which is overproduced in vascular diseases like atherosclerosis or diabetes-associated vasculopathy [6]. Analogously to the pro-inflammatory and pro-atherogenic adipocytokine IL$1 \beta$, the induction of iNOS by PBEF/NAMPT/visfatin required the sequential upstream activation of ERK 1/2 and NF- $\mathrm{KB}$ [8]. This latter transcription factor, which plays a pivotal role in the transcriptional regulation of multiple inflammation-related genes, is activated by ePBEF/ NAMPT/visfatin in another vascular cell type, the endothelial cell, resulting in the production of intercellular adhesion molecule-1, vascular cell adhesion molecule-1 and other mediators of inflammation [14, 15].

It is worth noting that the pro-inflammatory action of ePBEF/NAMPT/visfatin in HASMC was observed from a 


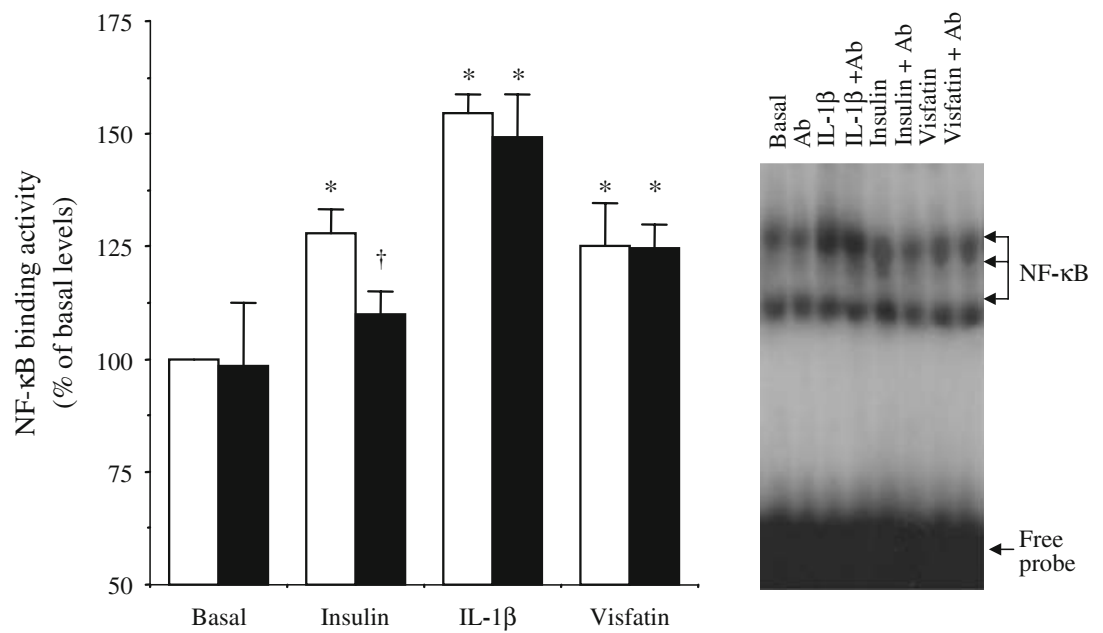

Fig. $3 \mathrm{NF}-\mathrm{kB}$ activation by ePBEF/NAMPT/visfatin in HASMC is not mediated by insulin receptor activation. HASMC were exposed for $18 \mathrm{~h}$ to insulin $(10 \mathrm{nmol} / \mathrm{l}), \mathrm{IL}-1 \beta(10 \mathrm{ng} / \mathrm{ml})$ or visfatin $(100 \mathrm{ng} / \mathrm{ml})$ and $\mathrm{NF}-\mathrm{KB}$ was determined by electrophoretic mobility shift assay (white bars). In parallel experiments, HASMC were pre-incubated for
$2 \mathrm{~h}$ with a commercial antibody $(\mathrm{Ab})$ that blocks the insulin receptor (black bars). A representative gel is shown on the right. Means \pm SEM of at least three independent experiments. ${ }^{*} p<0.05$ vs basal; $\dagger p<0.05$ vs matched treatment without antibody
Fig. 4 NAMPT activity mediates ePBEF/NAMPT/visfatininduced NF- $\mathrm{KB}$ activation and iNOS induction. HASMC were exposed to either the NAMPT activity product NMN $(100 \mu \mathrm{mol} / \mathrm{l})$ or visfatin $(100 \mathrm{ng} / \mathrm{ml})$ and $18 \mathrm{~h}$ later NF-KB activation (a) and iNOS induction (b) were determined. In another set of experiments, HASMC were treated for $18 \mathrm{~h}$ with visfatin $(100 \mathrm{ng} / \mathrm{ml})$ in the presence or absence of the NAMPT activity inhibitor APO866 (100 nmol/l), after which NF-KB activation (c) and iNOS levels (d) were determined. Representative gels are shown. Means \pm SEM of at least three independent experiments. $* p<0.05$ vs basal; $\uparrow p<0.05$ vs visfatin alone a
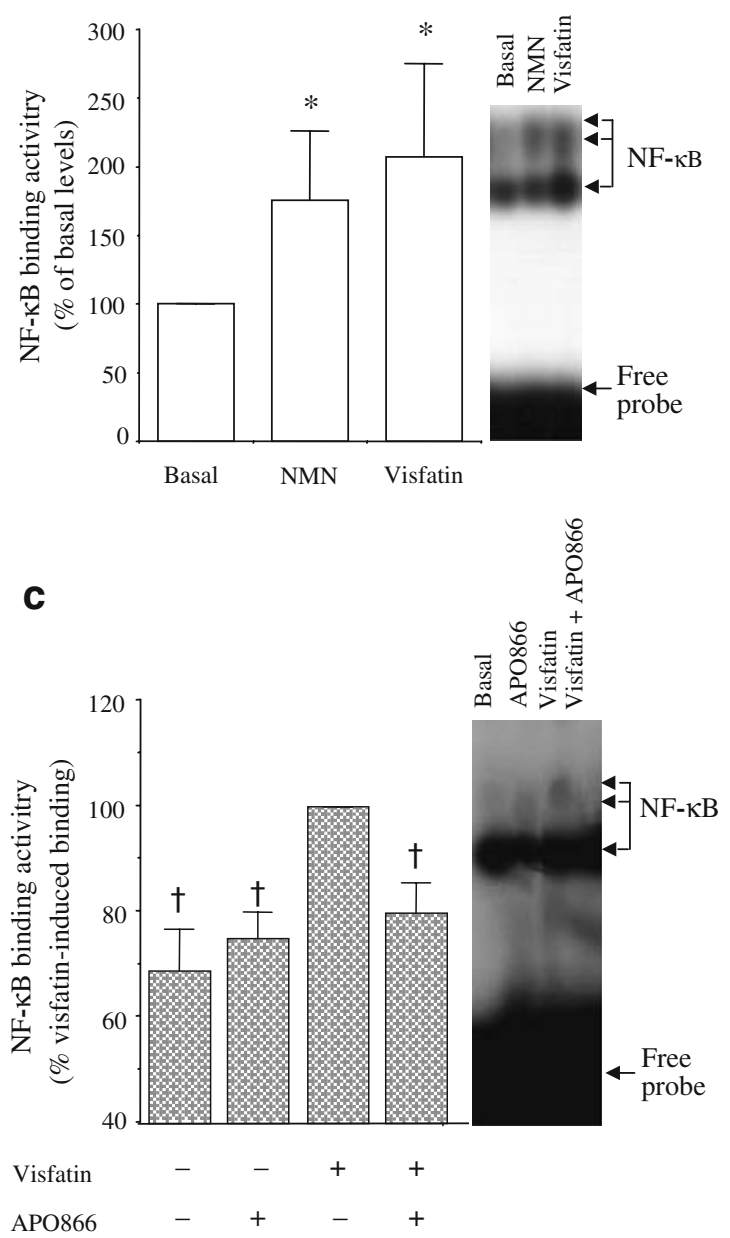

b $\mathrm{iNOS} \rightarrow$
$\alpha$-Tubulin $\rightarrow$

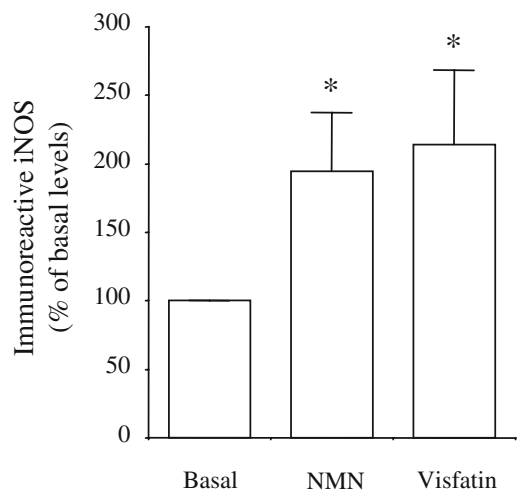

d
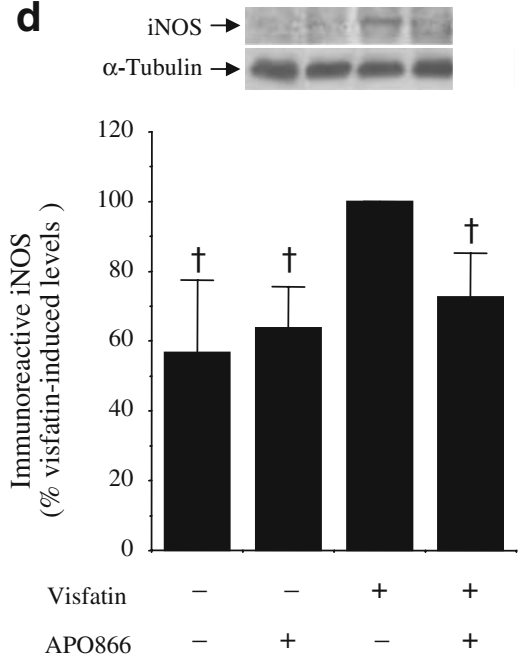
concentration of $50 \mathrm{ng} / \mathrm{ml}$. Although the number of studies measuring plasma levels of ePBEF/NAMPT/visfatin is still limited, circulating levels of ePBEF/NAMPT/visfatin of 40-60 $\mathrm{ng} / \mathrm{ml}$ have been reported in obese or type 2 diabetic patients, whereas their matched healthy controls showed lower levels $[2,3]$. Such elevated levels of plasma ePBEF/ NAMPT/visfatin have also been detected in patients with chronic kidney disease [5].

The current knowledge of the actions of PBEF/NAMPT/ visfatin in vascular smooth muscle cells is still limited. On the one hand, intracellular PBEF/NAMPT/visfatin has been reported to induce maturation [16] and to extend the lifespan of human vascular smooth muscle cells by activating members of the sirtuin family and restraining the accumulation of p53 [17]. On the other hand, ePBEF/ NAMPT/visfatin, released by different cells and tissues including the visceral and perivascular adipose tissue, can reach vascular smooth muscle and behave as a growth factor for this cell type [18]. In the present study, we have further identified ePBEF/NAMPT/visfatin as a proinflammatory factor for vascular smooth muscle cells.

A pro-inflammatory action of ePBEF/NAMPT/visfatin has been reported in non-vascular cell types and it has been related to different pathologies, such as arthritis, acute lung injury, inflammatory bowel disease or pre-term parturition $[19,20]$. In the context of cardiovascular diseases, ePBEF/ NAMPT/visfatin is seen as a new agent favouring vascular inflammation, a condition that plays a key role in the initiation and progression of atherosclerosis. Indeed, ePBEF/NAMPT/visfatin triggers the release of monocyte chemotactic protein-1, IL- 6 and IL- 8 and the activation of matrix metalloproteinases in human endothelial cells [21, 22]. Furthermore, an enhanced PBEF/NAMPT/visfatin content has been reported in human unstable carotid and coronary atherosclerotic plaques [23], as well as in the adipose tissue of patients with coronary artery disease, when compared with control individuals [24]. The ability of ePBEF/NAMPT/visfatin to activate and inflame human vascular smooth muscle demonstrated herein reinforces a potential pro-atherosclerotic action of the adipocytokine.

Although ePBEF/NAMPT/visfatin was initially claimed as an insulin mimetic acting through the insulin receptor and exhibiting glucose-lowering properties [1], such a statement was later on retracted and remains highly controversial [25]. On the one hand, Xie et al. [26] have reported that the regulation of glucose uptake, cell proliferation and type I collagen production in human osteoblasts by ePBEF/NAMPT/visfatin requires the activation of the insulin receptor. Furthermore, other cellular effects triggered by ePBEF/NAMPT/visfatin, including the secretion of IL- 8 and TNF- $\alpha$ by human peripheral blood mononuclear cells [23] and the uptake of glucose in rat mesangial cells [27], also seem to involve the insulin receptor. Other studies, however, have discarded a role for this receptor in mediating the actions of ePBEF/NAMPT/ visfatin in a wide range of cell types, including vascular cells, macrophages and pancreatic beta cells $[11,14,18$, 28]. The results presented herein do not support a role for the insulin receptor in mediating the pro-inflammatory action of ePBEF/NAMPT/visfatin in human vascular smooth muscle cells. Indeed, blocking the activation of the insulin receptor did not interfere with $N F-k B$ activation by ePBEF/NAMPT/visfatin or IL- $1 \beta$, and only abrogated the effects of insulin. The activation of NF- $\mathrm{kB}$ by insulin at pathophysiological concentrations $(10 \mathrm{nmol} /$ 1) [29] observed herein is in line with recent studies showing a vascular pro-inflammatory action of insulin through endothelial cell activation [30] and chemotaxis induction [31]. Nevertheless, our data indicate that insulin and ePBEF/NAMPT/visfatin exert their direct proinflammatory actions in vascular smooth muscle through distinct mechanisms.

In 2006, Revollo et al. [11] demonstrated that ePBEF/ NAMPT/visfatin regulates insulin secretion in pancreatic beta cells through its intrinsic NAMPT enzymatic activity. NAMPT converts nicotinamide to NMN, which is then transformed into NAD by nicotinamide/nicotinic acid mononucleotide adenylyltransferase [12]. In vertebrates, intracellular NAMPT plays an essential role in the biosynthesis of NAD from nicotinamide and therefore in the regulation of many cellular processes [12].

Here, we show that exogenous NMN mimicked the effects of ePBEF/NAMPT/visfatin on iNOS induction and $\mathrm{NF}-\mathrm{KB}$ activation in HASMC. In line with our observations, Wang et al. have recently reported that exogenous NMN, at the same concentration used in this study, mimics the proliferative action of ePBEF/NAMPT/visfatin in rat vascular smooth muscle [18]. Here, we demonstrate that extracellular NMN can also be considered as a proinflammatory agent for human vascular smooth muscle. Furthermore, the stimulatory action of ePBEF/NAMPT/ visfatin on iNOS and NF-KB in HASMC was abolished upon co-incubation with the NAMPT activity inhibitor APO866. Taken together, these results strongly suggest that extracellular NMN generation through NAMPT activity is on the basis of the pro-inflammatory action of ePBEF/ NAMPT/visfatin in human vascular smooth muscle. Such a mechanism of action also seems to account for the growth factor-like properties of ePBEF/NAMPT/visfatin in vascular smooth muscle cells [18]. NMN has been recently proposed to permeate mammalian cell membranes, possibly through NAD uptake mechanisms [11, 18, 32]. Therefore, the extracellular NMN generated trough NAMPT activity may enter the cell and then trigger the intracellular proinflammatory signals explored herein through mechanisms that remain to be elucidated. 
In conclusion, our findings identify ePBEF/NAMPT/ visfatin as an adipocytokine that can promote vascular smooth muscle cell inflammation. We propose that this effect is not mediated by the activation of the insulin receptor, but rather relies on the intrinsic NAMPT activity of the adipocytokine. We finally suggest that ePBEF/NAMPT/visfatin may have a direct role in vascular dysfunction and inflammation associated to metabolic disorders.

Acknowledgements We thank M. Martín Ayuso, Facultad de Medicina, Universidad Autónoma de Madrid, for collaboration in experimental work. This work was supported by grants from Ministerio de Educación y Ciencia (SAF2008-01291 and SAF200800942), Instituto de Salud Carlos III (RETICEF RD06/0013 and FIS PI061779), Comunidad de Madrid-Universidad Autónoma de Madrid (CCG07-UAM/BIO-1595), Fundación de Investigación Médica Mutua Madrileña, Instituto Danone and Sociedad Española de Farmacología/Laboratorios Almirall. T. Romacho is the recipient of a fellowship from Ministerio de Educación y Ciencia.

Duality of interest The authors declare that there is no duality of interest associated with this manuscript.

\section{References}

1. Fukuhara A, Matsuda M, Nishizawa M et al (2005) Visfatin: a protein secreted by visceral fat that mimics the effects of insulin. Science 307:426-430

2. Chen MP, Chung FM, Chang DM et al (2006) Elevated plasma level of visfatin/pre-B cell colony-enhancing factor in patients with type 2 diabetes mellitus. J Clin Endocrinol Metab 91:295-299

3. Filippatos TD, Derdemezis CS, Kiortsis DN, Tselepis AD, Elisaf MS (2007) Increased plasma levels of visfatin/pre-B cell colonyenhancing factor in obese and overweight patients with metabolic syndrome. J Endocrinol Invest 30:323-326

4. Takebayashi K, Suetsugu M, Wakabayashi S, Aso Y, Inukai T (2007) Association between plasma visfatin and vascular endothelial function in patients with type 2 diabetes mellitus. Metabolism 56:451-458

5. Yilmaz MI, Saglam M, Carrero JJ et al (2009) Normalization of endothelial dysfunction following renal transplantation is accompanied by a reduction of circulating visfatin/NAMPT. A novel marker of endothelial damage? Clin Transplant 23:241-248

6. Pacher P, Obrosova IG, Mabley JG, Szabó C (2005) Role of nitrosative stress and peroxynitrite in the pathogenesis of diabetic complications. Emerging new therapeutical strategies. Curr Med Chem 12:267-275

7. Peiró C, Matesanz N, Nevado J et al (2003) Glycosylated human oxyhaemoglobin activates nuclear factor-kappaB and activator protein-1 in cultured human aortic smooth muscle. Br J Pharmacol 140:681-690

8. Jiang B, Xu S, Hou X, Pimentel DR, Brecher P, Cohen RA (2004) Temporal control of NF-kappaB activation by ERK differentially regulates interleukin-1beta-induced gene expression. J Biol Chem 279:1323-1329

9. Haider DG, Schaller G, Kapiotis S, Maier C, Luger A, Wolzt M (2006) The release of the adipocytokine visfatin is regulated by glucose and insulin. Diabetologia 49:1909-1914

10. Taylor R, Soos MA, Wells A, Argyraki M, Siddle K (1987) Insulinlike and insulin-inhibitory effects of monoclonal antibodies for different epitopes on the human insulin receptor. Biochem J 242:123-129
11. Revollo JR, Körner A, Mills KF et al (2007) Nampt/PBEF/visfatin regulates insulin secretion in beta cells as a systemic NAD biosynthetic enzyme. Cell Metab 6:363-375

12. Formentini L, Moroni F, Chiarugi A (2009) Detection and pharmacological modulation of nicotinamide mononucleotide $(\mathrm{NMN})$ in vitro and in vivo. Biochem Pharmacol 77:16121620

13. Khan JA, Tao X, Tong L (2006) Molecular basis for the inhibition of human NMPRTase, a novel target for anticancer agents. Nat Struct Mol Biol 13:582-588

14. Kim SR, Bae YH, Bae SK et al (2008) Visfatin enhances ICAM-1 and VCAM-1 expression through ROS-dependent NF-kappaB activation in endothelial cells. Biochim Biophys Acta 1783:886895

15. Lee WJ, Wu CS, Lin H, Lee IT et al (2009) Visfatin-induced expression of inflammatory mediators in human endothelial cells through the NF-kappaB pathway. Int $\mathrm{J}$ Obes (Lond) $33: 465-472$

16. van der Veer E, Nong Z, O’Neil C et al (2005) Pre-B cell colonyenhancing factor regulates $\mathrm{NAD}+$-dependent protein deacetylase activity and promotes vascular smooth muscle cell maturation. Circ Res 97:25-34

17. van der Veer E, Ho C, O'Neil C et al (2007) Extension of human cell lifespan by nicotinamide phosphoribosyltransferase. J Biol Chem 282:10841-10845

18. Wang P, Xu TY, Guan YF, Su DF et al (2009) Perivascular adipose tissue-derived visfatin is a vascular smooth muscle cell growth factor: role of nicotinamide mononucleotide. Cardiovasc Res 81:370-380

19. Luk T, Malam Z, Marshall JC (2008) Pre-B cell colony-enhancing factor $(\mathrm{PBEF}) / \mathrm{visfatin}$ : a novel mediator of innate immunity. J Leukoc Biol 83:804-816

20. Mazaki-Tovi S, Romero R, Kusanovic JP et al (2008) Visfatin/ pre-B cell colony-enhancing factor in amniotic fluid in normal pregnancy, spontaneous labor at term, preterm labor and prelabor rupture of membranes: an association with subclinical intrauterine infection in preterm parturition. J Perinat Med 36:485-496

21. Liu SW, Qiao SB, Yuan JS, Liu DQ (2009) Visfatin stimulates production of monocyte chemotactic protein-1 and interleukin-6 in human vein umbilical endothelial cells. Horm Metab Res 41:281-286

22. Adya R, Tan BK, Chen J, Randeva HS (2008) Nuclear factorkappaB induction by visfatin in human vascular endothelial cells: its role in MMP-2/9 production and activation. Diabetes Care 31:758-760

23. Dahl TB, Yndestad A, Skjelland M et al (2007) Increased expression of visfatin in macrophages of human unstable carotid and coronary atherosclerosis: possible role in inflammation and plaque destabilization. Circulation 115:972-980

24. Cheng KH, Chu CS, Lee KT et al (2008) Adipocytokines and proinflammatory mediators from abdominal and epicardial adipose tissue in patients with coronary artery disease. Int J Obes 32:268-274

25. Fukuhara A, Matsuda M, Nishizawa M et al (2007) Retraction. Science 318:565

26. Xie H, Tang SY, Luo XH et al (2007) Insulin-like effects of visfatin on human osteoblasts. Calcif Tissue Int 80:201-210

27. Song HK, Lee MH, Kim BK et al (2008) Visfatin: a new player in mesangial cell physiology and diabetic nephropathy. Am J Physiol Renal Physiol 295:F1485-F1494

28. Li Y, Zhang Y, Dorweiler B et al (2008) Extracellular Nampt promotes macrophage survival via a non-enzymatic interleukin-6/ STAT3 signaling mechanism. J Biol Chem 283:34833-34843

29. Saad MF, Knowler WC, Pettitt DJ, Nelson RG, Mott DM, Bennett PH (1989) Sequential changes in serum insulin concentration 
during development of non-insulin-dependent diabetes. Lancet $1: 1356-1359$

30. Madonna R, Massaro M, Pandolfi A, Consoli A, De Caterina R (2007) The prominent role of p38 mitogen-activated protein kinase in insulin-mediated enhancement of VCAM-1 expression in endothelial cells. Int J Immunopathol Pharmacol 20:539-555
31. Kappert K, Meyborg H, Clemenz M et al (2008) Insulin facilitates monocyte migration: a possible link to tissue inflammation in insulin-resistance. Biochem Biophys Res Commun 365:503-508

32. Billington RA, Travelli C, Ercolano E et al (2008) Characterization of NAD uptake in mammalian cells. J Biol Chem 283:6367-6374 